\title{
ALÉM DAS GRADES: PERCEPÇÃO DE MULHERES ENCARCERADAS ACERCA DAS CONDIÇÕES DE SAÚDE
}

BEYOND BARS: IMPRISONED WOMEN'S PERCEPTION OF HEALTH STATUS

MÁS ALLÁ dE LAS REJAS: PERCEPCIÓN DE MUJERES ENCARCELADAS SOBRE EL ESTADO DE SALUD

Silmaria Bandeira do Nascimento ${ }^{1}$

Antônio Tiago da Silva Souza ${ }^{2}$

Marianne dos Santos Pereira ${ }^{3}$

João Rodrigo de Moura Carvalho ${ }^{4}$

Antonio Vladimir Félix da Silva 5

Palavras-chave:

Mulheres; Prisões; Cuidado;

Políticas Públicas.

Palabras clave:

Mujeres; Prisiones; Cuidado;

Políticas Públicas.

Submetido: $10 / 05 / 2019$

Aprovado:

11/10/2019

Autor(a) para

Correspondência:

Silmaria Bandeira do

Nascimento

Av. São Sebastião, 2819

Nossa Senhora de Fátima

Parnaíba (PI)

64202-020

E-mail:silmarianascimento@ hotmail.com

\section{RESUMO}

Este estudo teve por objetivo conhecer as condições de saúde de mulheres privadas de liberdade na penitenciária mista de Parnaíba (PI). Tratase de pesquisa qualitativa, descritiva e exploratória desenvolvida com 8 mulheres encarceradas em regime fechado. Foram realizadas entrevistas semiestruturadas para a coleta de dados, seguidas por análise de conteúdo temática. Os resultados indicaram 3 eixos temáticos que embasaram a análise e interpretação: a) "Minha saúde é razoável aqui dentro"; b) "Eles dão vacina na gente e passam exames"; e c) "A saúde do povo daqui só sabe quem vive". Constatou-se a existência de ações em saúde da mulher no sistema penitenciário, porém, de modo não regular, não programático e desarticulado. Conclui-se que há a necessidade de garantir que as políticas públicas voltadas às mulheres aprisionadas se mostrem efetivas, assim como se enfatiza a importância das equipes multidisciplinares de saúde definirem seu papel na assistência à saúde, de modo que possam fortalecer o vínculo entre os profissionais da saúde e os usuários dos serviços de saúde, com vistas a possibilitar que essas mulheres tenham participação ativa nos cuidados à sua saúde.

\footnotetext{
1. Psicóloga. Aluna de Especialização em Saúde da Família na Universidade Federal do Piauí (UFPI). Residente em Saúde da Família na UFPI. E-mail: silmarianascimento@hotmail.com ORCID: https://orcid.org/0000-0002$6035-106 \mathrm{X}$

2. Enfermeiro. Mestre em Enfermagem pela UFPI. Aluno de Graduação em Medicina na UFPI. E-mail: at.tiago@ hotmail.com ORCID: http://orcid.org/0000-0003-1904-1681

3. Aluna de Serviço Social na Cristo Faculdade do Piauí (CHRISFAPI). E-mail: marianne.dossantosp@gmail.com ORCID: https://orcid.org/0000-0002-2969-1891

4. Psicólogo graduado pela UFPI. E-mail: jrodrigo_moura@outlook.com ORCID: https://orcid.org/0000-00025426-471X

5. Pedagogo. Doutor em Ciências Psicológicas pela Universidade de Havana (Cuba). Professor na UFPI. E-mail: wladyfelix@hotmail.com 0RCID: http://orcid.org/0000-0003-3084-379X
} 


\section{ABSTRACT}

This study aimed to know the health status of women deprived of liberty in the mixed prison in Parnaiba, Piaui, Brazil. This is a qualitative, descriptive, and exploratory research conducted with 8 women in closed custody facilities. Semi-structured interviews were conducted for collecting data, followed by thematic content analysis. The results indicated 3 thematic axes that supported the analysis and interpretation: a) "My health status is acceptable in here;" b) "They vaccinate us and prescribe exams;" and c) "Only those who experience it know the health status in here." The existence of women's health actions was found in the prison system, but in a nonregular, non-programmatic, and non-comprehensive way. It is concluded that there is a need to ensure the public policies aimed at imprisoned women to be effective, as well as the importance that multidisciplinary health teams define their role in providing care is highlighted, so that they can strengthen the bond between health professionals and health service users, with a view to enabling these women to actively participate in their own health care.

\section{RESUMEN}

Este estudio tuvo como objetivo conocer el estado de salud de mujeres privadas de libertad en la prisión mixta en Parnaíba, Piauí, Brasil. Esta es una investigación cualitativa, descriptiva y exploratoria realizada con 8 mujeres reclusas en régimen cerrado. Se realizaron entrevistas semi-estructuradas para recopilar datos, seguidas de análisis de contenido temático. Los resultados indicaron 3 ejes temáticos que respaldaron el análisis y la interpretación: a) "Mi estado de salud es aceptable aqui"; b) "Nos vacunan y prescriben exámenes"; y c) "Solo quienes lo experimentan conocen el estado de salud aqui". Se constató la existencia de acciones en salud de la mujer en el sistema penitenciario, pero de manera no regular, no programática y desarticulada. Se concluye que es necesario garantizar que las políticas públicas dirigidas a las mujeres encarceladas sean efectivas, asi como se enfatiza la importancia de que los equipos de salud multidisciplinarios definan su papel en la atención de salud, para que puedan fortalecer el vínculo entre profesionales de la salud y usuarios de servicios de salud, con miras a permitir que estas mujeres participen activamente en sus propios cuidados de salud.

\section{INTRODUÇÃ O}

A população carcerária tem aumentado ao longo das últimas décadas no mundo inteiro. No Brasil, por conta disso, os presídios se encontram em precárias condições, que envolvem desde problemas estruturais relativos ao ambiente físico até a falta de vagas suficientes nas celas de aprisionamento. Tal quadro caracteriza violações aos direitos humanos fundamentais, em termos de alimentação, saúde, vestuário e relações sociais, impossibilitando o acesso a condições de saúde satisfatórias e gerando problemas de saúde pública ${ }^{1}$.

Em 1995, a Organização Mundial da Saúde (OMS) deu início um projeto chamado Saúde no Sistema Prisional, cujo objetivo era facilitar a interação entre o sistema prisional e os serviços de saúde pública, além de desenvolver ações voltadas à promoção da saúde por meio do cuidado integral nos presídios². No Brasil, que conta com a quarta maior população penitenciária do mundo (cerca de 725.000 presos, sendo que 689.000 estão em penitenciárias e 36.000 em delegacias carcerárias), o sistema penitenciário se encontra em um cenário de saúde alarmante ${ }^{3}$. Fragilidades no campo dos determinantes sociais, como trabalho, educação e lazer, entre outros, têm contribuído para o aumento da violência urbana e, consequentemente, a saturação do sistema carcerário, uma vez que a capacidade das prisões continua a mesma - a superlotação contribui para a propagação de inúmeras doenças, tanto físicas quanto psicológicas, entre os detentos. As penitenciárias femininas se inserem nesse contexto como instituições que agrupam uma população vulnerável a doenças infectocontagiosas s-5. $^{4}$.

0 perfil socioeconômico da população carcerária brasileira mostra que $55 \%$ dos detentos são jovens entre 18 e 29 anos, $64 \%$ são negros e $75 \%$ têm até - Ensino Fundamental incompleto. As mulheres somam cerca de 45.000 presas no Brasil, dentre elas, $62 \%$ estão envolvidas com o tráfico de drogas. A Lei de Execução Penal (LEP), em seu título II, capítulo II, seção III, art. 14, versa sobre a saúde da pessoa em restrição de liberdade, de modo que sejam garantidas ações preventivas e curativas. Para o desenvolvimento de tais ações, prevê-se que as equipes devem ser compostas por: a) 1 médico; b) 1 enfermeiro; c) 1 dentista; d) 1 psicólogo; e) 1 assistente social; f) 1 técnico em saúde bucal; e g) 1 técnico em enfermagem. E, caso o órgão não 
tenha aparelhamento para suprir as necessidades assistenciais, os cuidados em saúde devem ser proporcionados por outros dispositivos, com a devida autorização da direção institucional dos reeducandos em questão ${ }^{1}$.

Diante desse cenário, este estudo se propôs a conhecer a realidade das mulheres aprisionadas em Parnaíba (PI), a fim de identificar seu perfil e suas necessidades de saúde. Tal iniciativa de pesquisa possibilitou o aprimoramento da formação no âmbito de um programa de residência multiprofissional em Saúde da Família da Universidade Federal do Piauí (UFPI). Na área da psicologia, a escuta atenta das necessidades das mulheres presas e a valorização de suas falas e seus depoimentos evidenciam a preocupação com a qualidade das ações de saúde dentro do presídio, em linha com a integralidade da assistência preconizada pelo Sistema Único de Saúde (SUS).

\section{METODOLOGIA}

Trata-se de pesquisa qualitativa, descritiva e exploratória. A abordagem qualitativa é definida como metodologia que proporciona a produção de dados a partir de observações de pesquisas científicas com seres humanos, realizadas em locais nos quais os pesquisadores procuram estabelecer relações diretas com os participantes para compreender o cenário e os significados produzidos pelos sujeitos, ou seja, o foco não recai sobre a quantificação e mensuração de dados representativos de determinada realidade ${ }^{6}$.

0 local do estudo foi a penitenciária mista de Parnaíba, município situado no litoral piauiense. Essa instituição prisional foi inaugurada na década de 1990, em um espaço anteriormente ocupado por um mercado público, com área inferior a 3.000 $\mathrm{m}^{2}$, e capacidade para 200 detentos. Atualmente, recebe 650 internos (604 homens e 46 mulheres). Ressalta-se que essa quantidade varia de acordo com o fluxo diário de entrada e saída de detentos. Essa

\section{...conhecer a realidade das mulheres aprisionadas em Parnaíba (PI), a fim de identificar seu perfil e suas necessidades de saúde.}

instituição foi selecionada por conta de ser o único presídio local e por oferecer serviços de saúde às mulheres presas.

As participantes do estudo foram 8 mulheres em situação de cárcere. 0s critérios de inclusão foram: a) ser maior de 18 anos; e b) ter utilizado os serviços de saúde do sistema penitenciário. Já os critérios de exclusão foram: a) mulheres em regime semiaberto; e b) falta de consentimento para participar da pesquisa.

0s dados foram coletados por meio de entrevistas semiestruturadas: a) a primeira parte levantou informações acerca do perfil sociodemográfico das mulheres aprisionadas; b) a segunda parte levantou o estado geral de saúde no presídio, mediante questões norteadoras; e c) a terceira parte identificou as condições de saúde e como se viabilizam os cuidados em saúde e a garantia de direitos das detentas.

A categorização do material apontou 3 eixos temáticos que nortearam a análise e interpretação:

1. "Minha saúde é razoável aqui dentro";

2. "Eles dão vacina na gente e passam exames";

3. "A saúde do povo daqui só sabe quem vive".

A aplicação da entrevista ocorreu após a autorização da direção do presídio e o contato inicial com as mulheres, para o estabelecimento de vínculo e o convite para a participação na pesquisa, com exposição de seus objetivos, suas justificativas e sua relevância. Todas as participantes assinaram o termo de consentimento livre e esclarecido (TCLE) pertinente. 0 estudo foi aprovado pelo Comitê de Ética em Pesquisa da UFPI, sob o Parecer $n$. 3.221.606/2018, em conformidade com a resolução CNS n. 466/2012, que regulamenta a pesquisa com seres humanos no Brasil. A coleta de dados ocorreu em fevereiro e março de 2019.

Com duração média de 20 minutos, as entrevistas foram realizadas na sala do setor de psicologia da penitenciária, de acordo com a disponibilidade do serviço e durante os horários de banho de sol, sendo posteriormente transcritas e analisadas pelos autores deste artigo. Preservando a identidade das participantes, as falas foram submetidas a análise de conteúdo temática ${ }^{5}$.

\section{RESULTADOS E DISCUSSÃO}

As participantes da pesquisa tinham idades entre 25 e 72 anos. Quanto à escolaridade, 2 eram analfabetas, 5 tinham Ensino Fundamental incompleto e 1 tinha Ensino Superior incompleto. 
Em relação ao estado civil, 7 entrevistadas estavam solteiras e 1 estava civilmente casada.

Em termos de renda e trabalho, antes de dar entrada na penitenciária, 7 mulheres relataram ganhar menos de 1 salário mínimo, sendo que apenas 1 estava trabalhando e 6 já haviam trabalhado em algum momento de sua vida - 5 delas atuavam como diaristas, 1 como lavadeira e 1 como vendedora, com carteira assinada.

As informações das entrevistas acerca das condições de saúde das mulheres privadas de liberdade são apresentadas a seguir, sistematizadas e agrupadas nos 3 eixos temáticos adotados para a análise e interpretação.

\section{"Minha saúde é razoável aqui dentro"}

Nesta primeira categoria, buscou-se conhecer a percepção das mulheres aprisionadas sobre sua atual condição de saúde. Quando se perguntou qual era seu estado de saúde geral, 5 das 8 respondentes relataram ser razoável.

Um estudo demonstrou que o sistema prisional brasileiro apresenta péssimas condições de cuidados à saúde, principalmente quando se refere a questões de gênero ${ }^{7}$. As entrevistadas avaliam sua atual condição de saúde como razoável, apesar de considerarem o ambiente estressante, como indica a seguinte fala:

Minha saúde aqui tá boa, de vez em quando sinto uma dorzinha de cabeça, mas deve ser desse barulho ali, dos homens, que estressa a gente aqui. (Detenta 1 )

Percebe-se que o cenário de pesquisa contribui para aumentar o estresse das detentas, porém, para elas, isso não é considerado uma situação relevante em termos de saúde, limitando-se o conceito de saúde à ausência de doenças físicas.

$0 s$ depoimentos indicam que as mulheres desconhecem os riscos que o ambiente propicia à sua

\section{...o sistema prisional brasileiro apresenta péssimas condições de cuidados à saúde...}

saúde, o que é evidenciado quando perguntamos se alguma delas tinha doença(s) crônica(s):

Não... Só gastrite e pressão alta, mas tomo remédio, tem que dizer que a saúde é boa, né, pra ver se fica razoável, a comida aqui às vezes é sem gosto, mas não é pro bem da nossa saúde, né... (Detenta 2)

Além das participantes desconhecerem seus direitos de acesso à saúde e participação nos cuidados, nota-se a necessidade de aceitar a maneira como eles são postos em prática. 0 ideal é conhecer os determinantes sociais do processo saúdedoença da população apenada, para identificar suas necessidades e não priorizar as percepções dos profissionais da saúde - que, muitas vezes, mostramse contraditórias ${ }^{8}$.

\section{“Eles dão vacina na gente e passam exames"}

$\mathrm{Na}$ segunda categoria empírica, discute-se a percepção das mulheres acerca dos cuidados em saúde ofertados pelas equipes multidisciplinares dentro do presídio, bem como sua participação na produção desses cuidados.

Ações voltadas à prevenção de doenças e agravos e à promoção da saúde constituem importantes mecanismos para combater a proliferação de algumas doenças, mas os tratamentos voltados à população encarcerada ainda se mostram insuficientes e precários 9 . As falas apontaram algumas ações preventivas, mas também a passividade e fragilidade da assistência à saúde:

Eu fiz a consulta da pressão e aquela que fura o dedo. Tá com uns dois meses que a enfermeira me passou uns exames porque eu tava com umas dores de barriga, mas não sei que exame é... (Detenta 3)

A fala dessa participante parece não contemplar a promoção da saúde, principalmente naquilo que tange à transmissão de informações e à coparticipação nos cuidados mediante linguagem clara e objetiva. Isso se deve ao fato das ocorrências serem pontuais e resolvidas via ações ambulatoriais ou encaminhamentos (quando necessários) ${ }^{9}$.

0 cuidado integral a partir do trabalho em equipe se baseia no vínculo e na responsabilização, que diz respeito à ética e ao comprometimento profissional 


\section{...mostra-se necessário debater questões de gênero e discutir as políticas públicas...}

para buscar a resolutividade de modo conjunto ${ }^{10}$.

Eu tomei vacina quando cheguei aqui. Um dia desses me levaram para a doutora lá do centro, eu nem sei onde fica. Quem tá de encarregado é um sobrinho meu. Primeiro as enfermeiras olharam minha pressão, me deram conselhos pra eu me acalmar, aí o advogado me trouxe de volta com os agentes. (Detenta 4)

Vale salientar que, além dos profissionais de saúde, as participantes também incluíram suas famílias e seus advogados como agentes dos cuidados. Desse modo, mostra-se necessário debater questões de gênero e discutir as políticas públicas sob o olhar pactuado com outros setores, a fim de promover a garantia do direito à saúde postulado na Constituição Federal de 1988 pelos princípios da universalidade e da integralidade do SUS ${ }^{11}$.

Também se observou que as participantes não têm clareza acerca do papel assumido pelos profissionais da saúde, como demonstrado na seguinte fala:

0 médico de cabeça também ajuda a cuidar da minha saúde, ano passado eu não tomei vacina aqui, foi lá no posto de saúde porque eu ia fazer uma prevenção também. (Detenta 5)

Dentre as atribuições comuns aos profissionais da saúde temos a realização de atividades interdisciplinares e em equipe, integrando áreas técnicas e diferentes formações ${ }^{12}$.

\section{"A saúde do povo daqui só sabe quem vive"}

$\mathrm{Na}$ terceira categoria, as entrevistadas relataram algo que ainda não havia sido mencionado pelas demais - a falta de atenção aos casos relativos à saúde mental e aos serviços de saúde prestados indevidamente.
[...] A saúde do povo daqui só sabe quem vive. Essa colega aí, que você tá escutando, ela não deixa ninguém dormir. Faz mais de 15 dias que ela tá sem banho de sol, passa a noite gritando, os remédios dela não servem e eles não a levam pro psiquiatra passar outro. (Detenta 6)

Um estudo demonstra que o estabelecimento de vínculo razoável ou ruim dentro da prisão representa maior chance de desenvolvimento de agravos de saúde mental ${ }^{13}$. Além desse vínculo, a adaptação nas instituições prisionais provoca modificações subjetivas desde o início da internação, por meio do uso de roupas da instituição, do corte de cabelo obrigatório, da identificação por números, dos horários definidos pela administração, ou seja, a pessoa se transforma em objeto e ações que antes the conferiam autonomia são objetificadas em personagens prisionais ${ }^{14}$.

Às vezes a gente sente uma dor, eles pensam que é mentira, que a gente tá inventando. Sem falar também que tem agente aí que fica botando a maior banca pra nós não vermos os namorados. (Detenta 7 )

0 discurso das mulheres sobre a visita íntima demonstra que a instituição não vê esse momento como um direito das detentas - a sexualidade é oprimida e converte-se em alvo de abandono e julgamento ${ }^{15}$.

Essa realidade se mostra totalmente inadequada, segundo a política de mulheres privadas de liberdade, pois são necessários espaços específicos para a realização de visitas íntimas, de modo a garantir o vínculo familiar e a dignidade da mulher aprisionada ${ }^{16}$.

Quando se perguntou sobre o uso de algum método contraceptivo, a quantidade de filhos e a idade da primeira gestação, 5 das respondentes relataram não recorrer a nenhum dos recursos disponíveis e 5 informaram ter tido filhos entre os 14 e os 19 anos de idade.

Eu uso camisinha às vezes, já tenho muitos filhos, não quero mais, não. Meu último eu tive aqui. Fiz tudo aqui dentro, o pré-natal, as vacinas, mas ele só mamou dois meses. (Detenta 8) 
Vale destacar que os determinantes socioeconômicos, reprodutivos e sexuais relativos ao uso de contraceptivos se mostram significativos quanto ao conhecimento da forma correta de usar - medicamento e são relevantes para discutir a educação sexual e reprodutiva ${ }^{17}$.

Percebe-se que tais resultados corroboram os últimos relatórios sobre o sistema penal brasileiro, nos quais a maioria das mães é de perfil jovem; muitas vezes, elas são abandonadas pelo companheiro, sem condições adequadas de alimentação e de ambiência, de modo que mães e filhos permanecem em celas comuns e dividem espaço com as demais detentas ${ }^{18}$.

\section{CONCLUSÃO}

A partir das análises empreendidas, conclui-se que as condições de saúde das mulheres aprisionadas se mostram precárias em algumas situações e são garantidas em outras mediante os serviços ofertados pelas equipes multidisciplinares, ressaltando a importância das políticas voltadas tanto a mulheres privadas de liberdade quanto a egressas do sistema penitenciário.

No entanto, no que diz respeito à participação na produção dos cuidados de si, observou-se que as mulheres aprisionadas se mostram passivas e sujeitas à realidade prática dos serviços de saúde disponíveis no âmbito do sistema penitenciário, o que demonstra a rigidez e impessoalidade de um modelo verticalizado e hierarquizado, que não se pauta pela participação e pelo controle social.

Outro fato que chama atenção é a maneira como as mulheres nomeiam os membros da equipe de saúde dentro das instituições prisionais. Elas demonstraram dificuldade para definir a categoria profissional na equipe que presta os atendimentos de saúde. Não foi possivel evidenciar se essa dificuldade decorre das limitações técnicas e sociais na equipe, talvez caracterizadas por uma falha na definição de seus papéis enquanto profissionais da saúde ou se ela advém do tempo de aprisionamento das participantes, pois a maioria não tem muito tempo na prisão e isso pode dificultar o estabelecimento de vínculo entre o profissional da saúde e a paciente.

Nesse sentido, entende-se que se faz necessária maior participação por parte de toda a sociedade, para que as políticas públicas sejam efetivadas e garantam o direito à cidadania nas prisões brasileiras. Para tanto, gestores, profissionais da saúde e as

\section{...as mulheres apr isionadas se mostram passivas e sujeitas à realidade prát ica dos serviços de saúde disponiveis...}

famílias precisam lutar juntos para defender as mulheres encarceradas - que se veem em situação de vulnerabilidade além das grades, contando com uma rede de apoio fragilizada e fragmentada.

\section{CONTRIBUIÇÃO DOS AUTORES}

Silmaria Bandeira do Nascimento e João Rodrigo de Moura Carvalho contribuíram com a realização da pesquisa, o delineamento do estudo e a redação do manuscrito. Antônio Tiago da Silva Souza, Marianne dos Santos Pereira e Antonio Vladimir Félix da Silva contribuíram com o delineamento do estudo e a redação e revisão crítica do manuscrito.

\section{REFERÊNCIAS}

1. Almeida PRC, Soares RSC, Coura AS, Cavalcanti AL, Dutra MOM, Lima TMA. Condição de saúde de mulheres privadas de liberdade: uma revisão integrativa. Rev Bras Ciênc Saúde [serial on the internet]. 2015 [cited 2019 Dec 16];19(1):73-80. Available from: https://periodicos.ufpb.br/ojs2/index.php/rbcs/ article/view/23890/15053

2. Rio de Janeiro (Estado). Saúde e direitos humanos nas prisões [document on the internet]. 2001 [cited 2019 Dec 16]. Available from: http://www.dhnet. org.br/dados/manuais/dht/manual rj saude dh prisoes.pdf

3. Brasil. Levantamento nacional de informações penitenciárias [document on the internet]. 2018 [cited 2019 Dec 16]. Available from: http://www. justica.gov.br/news/ha-726-712-pessoas-presas-nobrasil/relatorio 2016 junho.pdf

4. Ribeiro SG, Lessa PRA, Monte AS, Bernardo EBR, Nicolau AI0, Aquino PS, et al. Perfil gineco-obstétrico de mulheres encarceradas no Estado do Ceará. Texto \& Contexto Enferm [serial on the internet]. 2013 [cited 2019 Dec 16];22(1):13-21. Available from: http://www.scielo.br/pdf/tce/v22n1/pt 02.pdf

5. Mourão LF, Oliveira LB, Marques ADB, Branco JG0, Guimarães MSO, Deus SEM. Promoção da saúde de mulheres encarceradas: um relato de experiência. 
Sanare (Sobral, Online) [serial on the internet]. 2015 [cited 2019 Dec 16];14(1):52-7. Available from: https://sanare.emnuvens.com.br/sanare/article/ view $/ 608$

6. Minayo MCS. 0 desafio do conhecimento: pesquisa qualitativa em saúde. São Paulo: Hucitec; 2007.

7. Silva Junior NGSE, Silva EBFL, Medeiros BG, Costa JNS. Revista íntima no sistema prisional paraibano: expressões da violência na subjetividade de familiares de presos. In: Oliveira AASO, Caniato $A M P C$, Fonseca DC, Almeida JAM, Prado Filho Kléber, Boarini ML, organizers. Psicologia social, violência e subjetividade. Florianópolis: Bosque; 2015. p. 2239.

8. Lima GMB. A vida de mulheres na prisão: legislação, saúde mental e superlotação em João Pessoa-PB [thesis]. João Pessoa: Fundação 0swaldo Cruz; 2013.

9. Carvalho LES, Sousa PCP, Veloso MV, Luz VLES, Feitosa VC. Percepção de detentos sobre a assistência à saúde em um presidio Estadual. Revista Interdisciplinar UNINOVAFAPI [serial on the internet]. 2016 [cited 2019 Jan 12];9(4):7988. Available from: https://revistainterdisciplinar. uninovafapi.edu.br/index.php/revinter/article/ view $/ 660$

10. Medeiros RHA. Uma noção de matriciamento que merece ser resgatada para o encontro colaborativo entre equipes de saúde e serviços no SUS. Physis (Rio J) [serial on the internet]. 2015 [cited 2019 Jan 5];25(4):1165-84. Available from: http:// www.scielo.br/pdf/physis/v25n4/0103-7331physis-25-04-01165.pdf

11. Assunção CHV. A saúde da mulher: a situação das encarceradas do Presídio Feminino de Florianópolis [monograph]. Florianópolis: Universidade Federal de Santa Catarina; 2010.

12. Santa Catarina (Estado). Atribuições da equipe de saúde no sistema prisional [document on the internet]. 2016 [cited 2019 Mar 14]. Available from: http://www.saude.sc.gov.br/ index.php/documentos/atencao-basica/sistemaprisional/10990-atribuicoes-da-equipe-de-saudeno-sistema-prisional/file

13. Constantino P, Assis SG, Pinto LW. 0 impacto da prisão na saúde mental dos presos do Estado do Rio de janeiro. Ciênc Saúde Colet [serial on the internet]. 2016 [cited 2019 Feb 7];21(7):2089-100. Available from: http://www.scielo.br/scielo.php?pid=S1413$81232016000702089 \&$ script $=s c i$ abstract\&tlng $=p t$

14. Brito DC, Lima AF. De bandidões a doentinhos: uma análise das identidades pressupostas numa instituição total. In: Oliveira AAS, Caniato AMP,

Fonseca DC, Almeida JAM, Prado Filho K, Boarini $M L$, organizers. Psicologia social, violência e subjetividade. Florianópolis: Bosque; 2015. p. 12345.

15. Guimarães MC. A problemática da visita intima no cárcere feminino: um estudo de caso sobre a Penitenciária Feminina Consuelo Nasser [dissertation]. Goiânia: Universidade Federal de Goiás; 2015.

16. Brasil. Política Nacional de Atenção às Mulheres em Situação de Privação de Liberdade e Egressas do Sistema Penitenciário. Brasília (DF): Ministério da Justiça; 2014.

17. Barros MAR, Penha JC, Galiza DDF. Relação dos condicionantes socioeconômicos, sexuais e reprodutivos quanto ao uso de método contraceptivo de presidiárias. Rev Enferm UFPE On Line [serial on the internet]. 2016 [cited 2018 Dec 20];10(12):4599605. Available from: https://pdfs.semanticscholar. org/7569/28da8cbb75d14b970289af50ebcd54b51a5f. $\underline{\mathrm{pdf}}$

18. Brasil. Política Nacional de Atenção Integral à Saúde das Pessoas Privadas de Liberdade no Sistema Prisional. Brasília (DF): Ministério da Saúde; 2014.
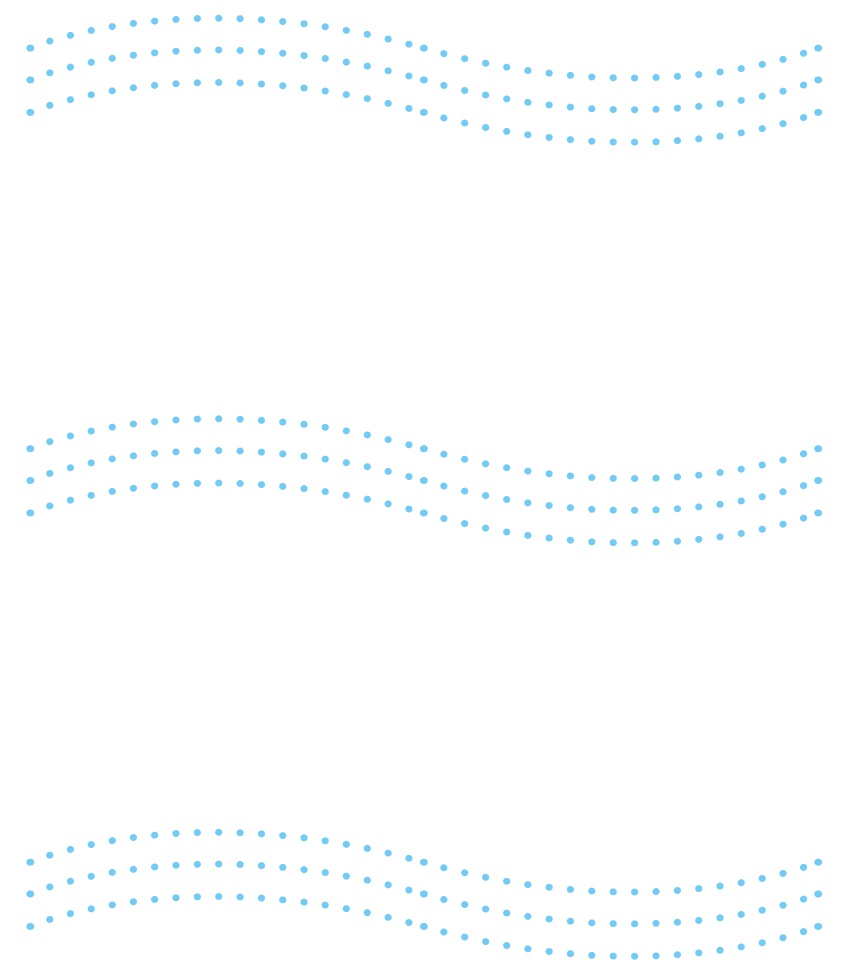